\title{
HIGH TIME RESOLUTION ANALYSIS OF SOLAR FLARES OBSERVED ON THE ESRO TD-1A SATELLITE
}

\author{
PETER HOYNG, JOHN C. BROWN*, GERARD STEVENS and H. F. VAN BEEK \\ Space Research Laboratory, University of Utrecht, The Netherlands
}

\section{Summary}

\section{(a) INSTRUMENT AND DATA REDUCTION}

The Utrecht Hard Solar X-Ray Spectrometer on board the ESRO TD-1A satellite (launch March 1972) is permanently Sun-pointed and measures the solar radiation between $30 \mathrm{keV}$ and $1000 \mathrm{keV}$, in 12 logarithmically spaced energy channels, with a continuous fine time resolution, viz. $1.2 \mathrm{~s}$ for the four lowest energy channels and $4.8 \mathrm{~s}$ for the rest. The detector has a $5 \mathrm{~cm}^{2} \mathrm{Cs} I(\mathrm{Na})$ crystal; counts due to particles are rejected and even during the largest solar flares saturation effects (e.g. pulse pile-up) are absent. For further details see Van Beek (1973), and Van Beek and De Feiter (1973).

The instrument has successfully operated during the two years' lifetime of the satellite and has observed a number of solar flares.

A method was developed that actually reconstructs the photon spectrum from the measured pulse height distribution and yet is much faster than the usual two parameter $\chi^{2}$-fit (Hoyng and Stevens, 1974). This permitted conversion of large amounts of pulse height distributions into photon spectra and thus full utilization of the $1.2 \mathrm{~s}$ time resolution. In addition, a single power law fit to the photon spectrum is routinely computed.

(b) OBSERVATIONAL MATERIAL; THICK TARGET ANALYSIS

A discussion is presented of our analysis of two smaller events, 1972, May 18, UT 1406, class 1B/M4 and 1972, August 7, UT 0252, class SB/M2, and the large event of 1972, August 4, UT 0620, class 2B/X5 (see Hoyng and Stevens, 1973, and Van Beek et al., 1973).

The May 18 event shows a regular single spike structure lasting $\sim 10^{2} \mathrm{~s}$, not unlike the spikes seen by Kane and Anderson (1970); the August 7 event was similar, except for its more detailed time structure. Above $100 \mathrm{keV}$ no flux was detected. The August 4 event is among the largest events ever recorded. The flare still emitted hard X-rays amply when data coverage ended after $21 \mathrm{~min}$ from the onset. In addition to rapid time variations the count rates appear to fluctuate periodically, the period increasing from some $20 \mathrm{~s}$ through $60 \mathrm{~s}$ to $120 \mathrm{~s}$. The photon spectra of the two small events

* On leave from Department of Astronomy, University of Glasgow, Scotland. 
are simple power laws with $\gamma$ increasing linearly (May 18) or constant (August 7) through the entire event, as also found by McKenzie et al. (1973) but contradicting Kane and Anderson's (1970) results. All authors however seem to find that towards the end of small events the spectrum softens. This feature, together with the detailed (spiky) structure of the time profile itself, are the main arguments for the existence of continuous electron acceleration.

The spectra of the August 4 event, detectable up to $\sim 400 \mathrm{keV}$, are not simple power laws. They have a break, not necessarily sharp, around $\sim 60 \mathrm{keV}$ after which the spectrum steepens by $\Delta \gamma \sim 1$. (In the single power law fits, $\gamma(t)$ decreases towards the end of the event). This break (cf. also Frost, 1969) is probably too large to be interpreted as an anisotropy effect (cf. Petrosian, 1973) and so should be present in the spectrum of the X-ray emitting electrons. However, as long as no good theoretical explanation for pure power laws exists, observation of a power law with a break must not be considered as more peculiar than a simple power law itself (cf. the cosmic ray spectrum). Using the single power law fits, we determined for all three events with full time resolution the thick target parameters $F_{25}$ and $P_{25}$, being the total required number flux and energy flux of fast electrons $\geqslant 25 \mathrm{keV}$ into a target region (Brown, 1971). Table I summarizes typical values.

\section{TABLE I}

Total required number and energy flux of electrons $\geqslant 25 \mathrm{keV}$ in a thick target $\mathrm{X}$-ray source

\begin{tabular}{lrc}
\hline & $\begin{array}{c}\text { Smaller event } \\
\left.\text { (lasting } 10^{2} \mathrm{~s}\right)\end{array}$ & $\begin{array}{l}\text { Large event } \\
\left.\text { (lasting } 10^{3} \mathrm{~s}\right)\end{array}$ \\
\hline Mean $F_{25}\left(\mathrm{~s}^{-1}\right)$ & $0.5-1.0 \times 10^{36}$ & $4 \times 10^{36}$ \\
$\int F_{25} \mathrm{~d} t$ & $4 \times 10^{37}$ & $5 \times 10^{39}$ \\
$\int P_{25} \mathrm{~d} t(\mathrm{erg})$ & $2 \times 10^{30}$ & $2 \times 10^{32}$ \\
Mean $n_{0} N_{25}\left(\mathrm{~cm}^{-3}\right)$ & $3-8 \times 10^{45}$ & $3.5 \times 10^{46}$ \\
\hline
\end{tabular}

The interpretation of these numbers poses great problems: the energy put into fast electrons about equals the total flare energy spent and the total number of electrons ever accelerated is about equal to the number of electrons in the whole flare, taking some reasonable density $\left(10^{10-11} \mathrm{~cm}^{-3}\right)$. Moreover these numbers are lower limits in the sense that one could well extrapolate below $25 \mathrm{keV}$ and/or the target might be thin!

By using reasonable inflow velocities into some flare region we find that in particular $F_{25}$ is too high. We argue that the only practical way to bring $F_{25}$ down is by accelerating an electron not once but many times. In this case $F_{25}$ has no meaning and from the data one derives the nonthermal emission measure $n_{0} N_{25}=$ ambient density $\times$ total number of fast electrons $\geqslant 25 \mathrm{keV}$ at the given time. One could term this a thick target with containment. There is however no way around the large values 
of $\int P_{25} \mathrm{~d} t$ and we have to face the situation that nearly all flare energy is channeled through fast electrons into other forms. An interesting new feature of the data is the marked correlation of $\gamma$ and $F_{25}$ in the August 4 event. During the last 7 min (decay phase) the correlation is particularly good and $\gamma$ and $F_{25}$ decrease monotonically with time. This could indicate the slow collisional decay of a large, low density cloud. From elementary considerations we find a density $n_{0} \sim 10^{8} \mathrm{~cm}^{-3}$ and a volume $V \sim 10^{33}$ containing $\sim 10^{38}$ fast electrons. These electrons could be due to escape of a small fraction of the total number available, viz. $5 \times 10^{39}$ (Table I).

\section{(c) FOURIER ANALYSIS}

Power spectra have been computed of the raw count rates of channel 2, $F_{25}$ and $\gamma$ for the three events (the assistance of Drs R. Rutten and G. Geytenbeek is gratefully acknowledged). The results can be summarized as follows:

(i) The power spectrum of the raw count rates of channel 2 of the August 4 event shows significant periodicities at 120,60 and $33 \mathrm{~s}$. The same periodicities are present in the power spectra of $F_{25}$ and, to a lesser degree, of $\gamma$. No periodicities were found in the two other events.

(ii) In all three events, the power spectrum of the raw count rates of channel 2 shows an average high frequency power level significantly above the level expected from Poisson noise alone. We drew the conclusion that the solar flare must have intrinsic white noise in its emission at all periods from $15 \mathrm{~s}$ down to the Nyquist period, $2.4 \mathrm{~s}$.

The periodicities in the August 4 event must correspond to coherent changes of the whole flare region. Directly from the time profile we infer physical timescales $\tau=|(1 / c)(\mathrm{d} c / \mathrm{d} t)|^{-1}, c=$ count rate, for these changes and find $\tau \gtrsim 10 \mathrm{~s}$ for the large event and $\tau \gtrsim 2 \mathrm{~s}$ (non periodic) for the small events - so that neither case shows changes in the source as a whole in times down to $1.2 \mathrm{~s}$.

\section{References}

Brown, J. C.: 1971, Solar Phys. 18, 489.

Frost, K.: 1969, Astrophys. J. 158, L159.

Hoyng, P. and Stevens, G. A.: 1973, Proceedings First European Astronomical Meeting, Athens 1972, vol. 1, 97.

Hoyng, P. and Stevens, G. A.: 1974, Astrophys. Space Sci. $27,307$.

Kane, S. R. and Anderson, K. A.: 1970, Astrophys. J. 162, 1003.

McKenzie, D. L., Datlowe, D. W., and Peterson, L. E.: 1973, Solar Phys. 28, 175.

Petrosian, V.: 1973, Astrophys. J. 186, 291.

Van Beek, H. F.: 1973, Ph.D. Thesis, Utrecht.

Van Beek, H. F. and de Feiter, L. D.: 1973, Proceedings First European Astronomical Meeting, Athens 1972, vol. 1, 103.

Van Beek, H. F., Hoyng, P., and Stevens, G. A.: 1973, in H. E. Coffey (ed.), Collected Data Reports on the August 1972 Solar-Terrestrial Events, Report UAG-28, part II, 319. 